\author{
GERNOT KOCHER \\ Institut für Österreichische Rechtsgeschichte und Europäische \\ Rechtsentwicklung Karl-Franzens-Universität Graz
}

\title{
65-jähriges Jubiläum von Ludwig Karničar
}

(Laudatio, Graz, 4. oktober 2014)

Hohe Festversammlung, lieber Ludwig!

Als die Babylonier - so berichtet das Buch Genesis 11,7 - beschlossen, einen bis in den Himmel reichenden Turm in Babel zu errichten, erregten sie damit den Unmut des Herrn - seine Reaktion kam postwendend mit der Aufforderung: „Auf, steigen wir hinab und verwirren wir dort ihre Sprache, so dass keiner mehr die Sprache des anderen versteht." Leider hat die nachfolgende Erlösung der Menschheit durch Gottes Sohn diese Strafmaßnahme nicht beseitigen können. Daraus ergibt sich dann natürlich zwangsläufig die Frage, wie denn mit der Klärung der Sündenfrage am Tage des Jüngsten Gerichtes verfahren wurde.

Man wird wohl von der - mangels Information vielleicht sogar unwiderlegbaren - (Rechts)-Vermutung ausgehen müssen, dass Gott „allsprachlich“ zu denken ist. Damit wäre für das Jenseits die Sprachenfrage wohl gelöst, aber im Diesseits hat sich am babylonischen Sprachengewirr nichts geändert, somit ist wohl klar, dass die Translationswissenschaften beziehungsweise die Sprachwissenschaften beim Turmbau von Babel ihren Ausgangspunkt haben - und da kommen wir nun endlich zu unserem Ludwig, der sich unter den seit damals wirkenden „Sprachmittlern“ eine hervorragende Position erworben hat.

Bevor ich nun näher den Strahlenkranz um Ludwigs Kopf näher analysiere, will ich kurz erläutern (was sich viele vielleicht schon gefragt haben), wieso ein Rechtshistoriker dazu kommt, hier auf fremdem Territorium aufzutreten. Die einfachste Antwort wäre, ich wurde eben eingeladen. Aber alles hat seine Hintergründe, und diese beginnen schon beim Großvater, der vergeblich versuchte, mir Slowenisch beizubringen und führen über Projektmitarbeiter aus Südkärnten (Ludmannsdorf, Rosental) direkt zu einem Sprachkurs bei Ludwig und in der Folge zur engen wissenschaftlichen Zusammenarbeit mit den rechtswissenschaftlichen Fakultäten in Maribor und Ljubljana. Das mit dem Sprachkurs ist jetzt schon fast dreißig Jahre her und wer, so frage ich, hätte damals gedacht, dass ich jemals in die Lage kommen würde, für die Sprachwissenschaften, aber insbesondere für die Slowenistik etwas zu tun?

Nun, die Wege des Schicksals sind oft seltsam und so stehe ich hier - mehr oder weniger gerechtfertigt als Außenstehender (Altdekan) - um den wissen- 
schaftlichen Weg einer Person zu beleuchten. Vielleicht ist der Blick von Außen sogar besser, denn die Innensicht ist bei allen ja vorhanden, man weiß ja, mit welchen hervorragenden Leistungen Ludwig zum Überleben der Slowenistik in Graz beigetragen hat.

Wenden wir uns nun einmal der Person zu. Er ist - um einen heute sehr gängigen Ausdruck zu verwenden - eine ,geerdete“ Persönlichkeit, 1949 als ältestes von acht Kindern in eine Bergbauernfamilie in Ebriach/Obirsko hineingeboren und mit der zweisprachigen Volksschule und dem Bundesgymnasium für Slowenen in Klagenfurt wurde auch die Grundlage für seinen späteren Beruf geschaffen. Musische Begabung, die wohl Hand in Hand geht mit Sprachbegabung, war ihm in die Wiege gelegt, wie sein späterer Lebensweg klar beweist. Gleich nach der mit Auszeichnung abgelegten Matura verschlug es ihn mit dem American Field Service nach Amerika und nach dem Präsenzdienst studierte er Russisch, Spanisch und Südosteuropäische Geschichte, begleitet von Studienaufenthalten in Moskau, Ljubljana und Zadar.

Auf die Promotion 1979 folgte fast unmittelbar die Integration in die universitäre Lehre und dann in den Wissenschaftsbetrieb mit der Habilitation.

Die Dissertation, abgeschlossen 1979, mit dem Titel „Der Obir-Dialekt in Kärnten" lässt schon den zukünftigen wissenschaftlichen Weg erkennen, der vornehmlich von zwei akademischen Lehrern, Stanislaus Hafner und Erich Prunč begleitet worden war und in dem es um die Erfassung und damit auch Erhaltung der vom Aussterben bedrohten Kärntner slowenischen Dialekte geht ein Problem, dass er erst kürzlich in einem Vortrag mit dem Titel „Verstummen die slowenischen Dialekte in Kärnten" klar angesprochen hat.

Die Österreichische Akademie der Wissenschaften unterstützte dieses Projekt zur Dialekterfassung, das wohl unendliche Arbeit bedeutete, die letztlich an ihm und den zeitweilig zur Verfügung stehenden Mitarbeitern hängen blieb. Mein persönlicher Eindruck, gewonnen als ich einmal wegen einer Frage zum Kurs bei ihm im Institut (noch in der Mozartgasse) war, wurde von einer fast unüberschaubaren Masse von Karteikärtchen geprägt - selbst für mich überwältigend, obwohl ich damals schon gewohnt war, mit tausenden Bildern und Belegstellen zu arbeiten. Das Ergebnis lässt sich durchaus sehen, der siebente Band, der bereits beim Buchstaben M angelangt ist, erschien 2012. Dass es vermutlich nicht mehr weiter gehen soll, ist eine wirklich tragische Entwicklung, die wieder einmal belegt, welche Wertschätzung geisteswissenschaftlicher Arbeit entgegengebracht wird - aber, die Hoffnung, lieber Ludwig, stirbt zuletzt. Aus dieser Arbeit sind eine ganze Reihe sprachwissenschaftlicher Untersuchungen hervorgegangen: Ich nenne nur zwei, über die „Phraseologie der Tierwelt" (1993) oder über „Diatopische Synonyme für Kartoffel“ (2007), die auch wieder seine „Erdverbundenheit“ bezeugen.

Das sprachwissenschaftliche Herzstück seiner Arbeit wird rundum ergänzt durch historische Recherchen, die sich mit den Pionieren der Slowenistik in den altösterreichischen Ländern und damit auch mit der universitären Pflege dieser Disziplin beschäftigen - so etwa über ,Janez Nepomuk Primic in slovenščina v Gradcu“ (2002), „Slovenistika v Gradcu: včeraj, danes, jutri“ (2005), „Die 
illyrischen Provinzen und die erste slowenische Lehrkanzel in Graz" (2009). In diese Linie gehört auch die Herausgabe der „Materialien zur Geschichte der Slawistik in der Steiermark“, gemeinsam mit Erich Prunč 1987.

Wissenschaft zu betreiben, ohne organisieren zu können, ist nur eine halbe Sache. Ludwig hat auch hier seine Qualitäten unter Beweis gestellt, wobei die Vielseitigkeit des Ertrages dieser Veranstaltungen zu betonen ist: Sprachwissenschaft, Sprachgeschichte und Kulturgeschichte profitierten davon. $\mathrm{Zu}$ nennen sind hier das 7. Slowenistische Symposium (2008), die 2 Tagungen zu „Graz und Slowenen“ (2010 und 2014), dann „200 Jahre weltälteste Lehrkanzel für Slowenisch“ (2011) und eine „Akademie zum 100. Todestag von Prof. Karel Štrekelj (2012). Darüber hinaus diente Ludwig Karničar der Wissenschaft auch durch die Mitarbeit in wissenschaftlichen Vereinigungen, so in der Balkankommission der Österreichischen Akademie der Wissenschaften und im Leitungsgremium des Slowenischen Wissenschaftsinstitutes in Wien, einer außergewöhnlichen Einrichtung zur Förderung der grenzübergreifenden Forschung, für deren Erhalt Ludwig gemeinsam mit Vincenc Rajšp wie ein Löwe gekämpft hat, Kämpfe, an denen ich auch persönlich großen Anteil genommen habe, da es sich hier um eine dem Nutzen beider Staaten dienende Institution handelte, die sich außerdem auf höchstem europäischem Niveau bewegte.

Das Bild von Ludwig Karničar wäre unvollständig, würde man die anfangs schon erwähnte musische Komponente außer Acht lassen: Zahlreiche Publikationen und Audioeditionen - diese gemeinsam mit Engelbert Logar - beschäftigen sich mit dem Liedgut der Slowenen in Südkärnten, wobei die Achse zu den singenden Gebrüdern Smrtnik auch bei verschiedenen Tagungen einen Hörgenuss vermittelte, an dem die schöne, solo locker eine große Kirche füllende Stimme von Ludwig nicht unbeteiligt war.

Kehren wir zum Schluss noch einmal an den Ausgangspunkt, den Turmbau zu Babel mit dem darauf folgenden Sprachengewirr zurück - die Verbindung zu Ludwig Karničar ist ganz offenkundig: Seine über Jahrzehnte dauernde Dolmetschtätigkeit in einer Zeit des „Alpen-Adria-Aufbruches“, wo es um den Bau von geistigen, politischen und wirtschaftlichen Brücken ging, ist im Sinne einer Ausgleichshandlung gegenüber dem göttlichen Urteilsspruch zu sehen. Dazu gehört aber auch das gemeinsam mit Paul Apovnik geschaffene deutsch-slowenische und slowenisch-deutsche Wörterbuch der Rechts- und Wirtschaftssprache $(1989$ /1996), damit knüpfte man an Wörterbuchpioniere des 19. Jahrhunderts, wie Matej Cigale, Janko Babnik und Franz Miklosich an.

Insgesamt, lieber Ludwig, war der Ausdruck „Strahlenkranz“ für Deine vielfältigen Leistungen für die Slowenistik genausowenig übertrieben, wie die Bezeichnung als „erdverbunden“. Du hast die Heimatverbundenheit zur Basis Deines Berufes gemacht und damit der Sprachwissenschaft allgemein, aber auch an der Universität Graz einen großen Dienst erwiesen, wofür wir Dir alle, das Institut, die Fakultät und die Universität danken. Wir wünschen Dir für Deinen neuen Lebensabschnitt weiterhin viel Schaffensfreude und die dafür notwendige Gesundheit. 